\title{
Conversão catalítica de alquilaromáticos sobre catalisadores micro-mesoporosos em reator gás-sólido de leito fixo visando à obtenção de olefinas
}

Catalytic conversion of alkylaromatic over micro-mesoporous catalysts in the gas-solid fixed bed reactor in order to produce olefins

\author{
A.C. B. Rêgo ${ }^{1 *}$; E. V. Nascimento ${ }^{2}$; T. G. S. Nascimento ${ }^{2}$; A. M. Garrido \\ Pedrosa $^{3} ;$ M. J. B. Souza ${ }^{1,2}$
}

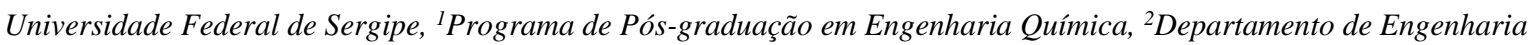
Química, ${ }^{3}$ Departamento de Química, 49100-000, São Cristóvão-Sergipe, Brasil

*anacarolrego@gmail.com

(Recebido em 14 de março de 2016; aceito em 22 de abril de 2016)

\begin{abstract}
O objetivo deste trabalho foi estudar a síntese e caracterização de catalisadores micro-mesoporosos e sua aplicação na reação modelo de conversão de isopropilbenzeno em microreator catalítico de leito fixo com fluxo contínuo visando à obtenção de olefinas. Catalisadores micro-mesoporosos do tipo HZSM-5/MCM-41e La/HZSM-5/MCM-41foram sintetizados através do método mecanosíntese e impregnação por via úmida, respectivamente. Os catalisadores foram caracterizados por difração de raios-X e através de reações com molécula modelo. Isopropilbenzeno (cumeno) foi usado como molécula modelo e a conversão em olefinas foi avaliada através da formação de propeno. Os difratogramas de raios-X indicaram que os catalisadores sintetizados apresentam os picos característicos da estrutura microporosa (zeólita ZSM-5) e a estrutura mesoporosa (MCM-41). Para os catalisadores contendo lantânio, picos devido à presença de óxido de lantânio também foram identificados. Os resultados indicaram que o catalisador compósito La/HZSM-5/MCM-41 foi o mais promissor na conversão do cumeno e produção de propeno.
\end{abstract}

Palavras-chave: HZSM-5, MCM-41, Conversão catalítica, Alquilaromáticos, Propeno

The aim of this work was to study the synthesis and characterization of micro-mesoporous catalysts and their application in model reaction of conversion of isopropylbenzene in fixed bed continuous flow catalytic microreactor in order to produce oefins. The micro-mesoporous catalysts of HZSM-5/MCM-41 and La/HZSM5/MCM-41 were synthesized by the mechanosynthesis and wet impregnation, respectively. These catalysts were characterized by X-rav diffraction, and through reaction with model molecule. Isopopylbenzene (cumene) was used as model molecule and the conversion to olefins was measured by propylene formation. The X-ray diffractograms indicated that the synthesized catalysts exhibit characteristic peaks of the microporous structure (ZSM-5) and mesoporous structure (MCM-41). For catalysts containing lanthanum, peaks due to the presence of lanthanum oxide were also identified. The results indicated that the composite catalyst La/HZSM-5/MCM-41 was the most promising in the conversion of cumene and propene production.

Keywords:HZSM-5, MCM-41, Catalytic Conversion, Alkylaromatics, Propene

\section{INTRODUÇÃO}

Processos de craqueamento catalítico em leito fluidizado são processos muito importantes no contexto de uma refinaria, onde as cargas de petróleo pesado utilizados são de baixo valor agregado, como por exemplo, o gás óleo de vácuo $(\mathrm{GOV})$, os quais são otimizados em produtos de alto valor agregado, principalmente gasolina, GLP e olefinas $\mathrm{C}_{3} / \mathrm{C}_{4}$ [1]. As unidades de FCC podem atingir capacidades de processamento muito elevadas, em alguns casos ultrapassando cerca de 6 milhões de toneladas por ano [2].

Nestes processos, a quebra das moléculas ocorre devido à ação da temperatura e as propriedades catalíticas do meio, e sendo assim a escolha do catalisador apropriado pode determinar o sucesso do processo de craqueamento. Logo, desenvolver catalisadores de craqueamento mais eficientes é de suma importância para o aumento da oferta de frações leves de petróleo [2, 3].

Catalisadores comerciais de FCC são tipicamente compostos de uma zeólita ácida microporosa dispersa em uma matriz amorfa de sílica-alumina mesoporosa, um diluente do tipo caulinita, um ligante e alguns aditivos, como por exemplo, um elemento terra rara como lantânio ou cério $[2,4]$. 
Em processos de craqueamento catalítico se utiliza uma zeólita de poros largos tendo uma boa estabilidade térmica e hidrotérmica. É normalmente inserido um elemento terra rara por troca iônica $[2,5]$. As propriedades catalíticas são influenciadas pela composição da estrutura da zeólita $(\mathrm{Si} / \mathrm{Al})$ e pela presença de espécies incluindo contra-íons. Em geral, pequenos cristais da zeólita Y são preferidos, a fim de reduzir problemas de difusão, aumentar a estabilidade e proporcionar seletividade catalítica ótima. Nos últimos anos tornou-se comum incluir nos catalisadores de FCC uma pequena quantidade de ZSM-5 para melhorar a seletividade [6].

Um dos componentes catalíticos utilizados em processos de conversão catalítica é a zeólita ZSM-5, caracterizada por apresentar um alto grau percentual de silício (relação Si/Al compreendida entre 15 e infinito) [7]. A ZSM-5 é utilizada em processos de craqueamento catalítico para melhorar a octanagem da gasolina e também produção de olefinas, promovendo uma maior qualidade, sendo composta em sua estrutura por um sistema de canais em zig-zag com sistemas de poros de canais interligados bidimensionais com janelas de 5-6 Å (Figura 1) [7, 8].

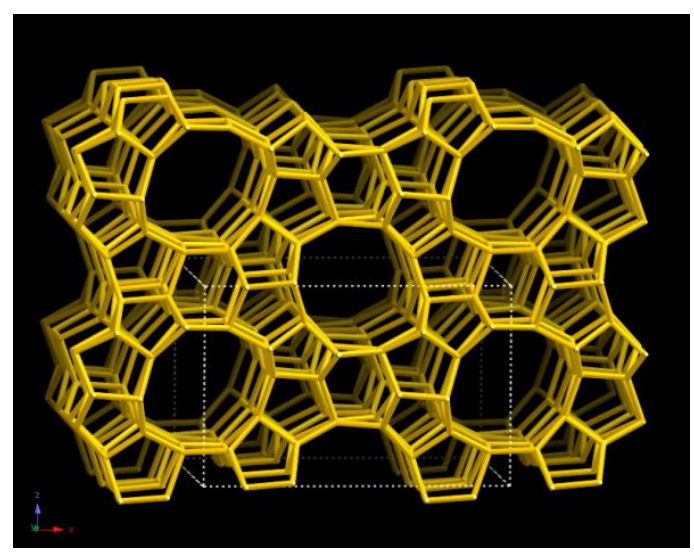

Figura 1: Estrutura da zeólita ZSM-5 [9].

Os materiais mesoporosos da família M41S (Figura 2), constituídos pelo MCM-41, MCM-48 e MCM-50, possuem diâmetro de poro maior que as zeólitas, promovendo maior difusividade de moléculas volumosas. Porém a baixa estabilidade térmica e acidez que estes materiais apresentam faz com que eles não sejam escolhidos para substituir as zeólitas em diversos processos [10].
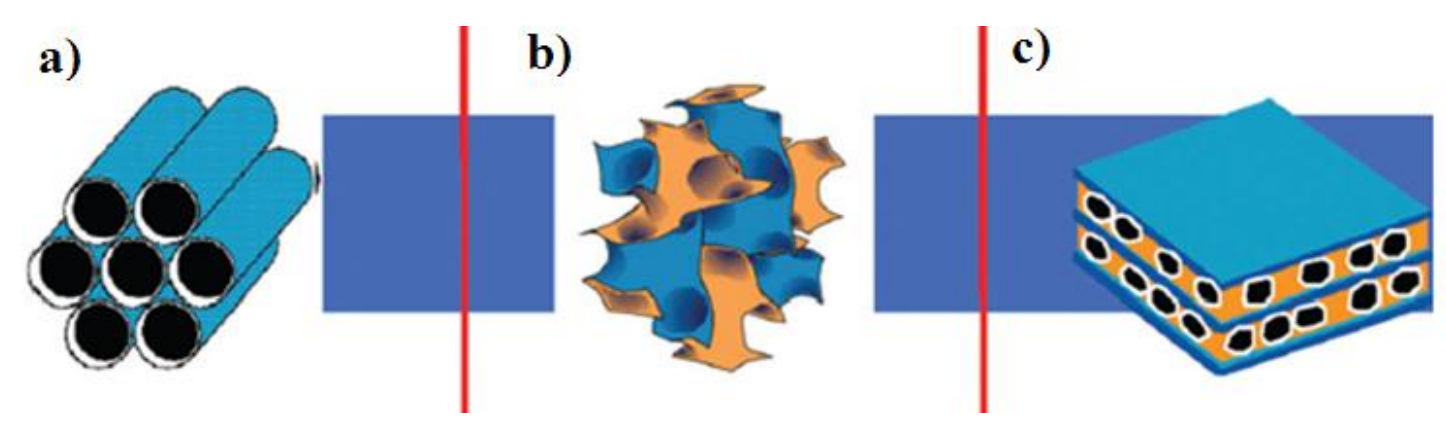

Figura 2: Ilustração das estruturas mesoporosas da família M41S : a) MCM-41;b) MCM-48; c) MCM-50, adaptado de [10].

O presente trabalho tem como objetivo investigar o desenvolvimento, caracterização e aplicação de catalisadores heterogêneos micro-mesoporosos do tipo HZSM-5/MCM-41 e La/HZSM-5/MCM-41 na conversão catalítica de alquilaromáticos visando à obtenção de olefinas.

A combinação dos dois tipos de estrutura (microporosa zeolítica e mesoporosa) pode gerar um catalisador compósito que apresenta propriedades atrativas para usos em catálise, como é o caso da conversão catalítica de alquilaromáticos visando à produção seletiva de olefinas. 


\section{MATERIAL E MÉTODOS}

\section{1- Síntese}

A síntese da zeólita microporosa HZSM-5 foi realizada através da calcinação do produto comercial CBV8014 que representa a amostra ZSM-5 amoniacal ( $\left.\mathrm{NH}_{4} \mathrm{ZSM}-5\right)$. As condições de calcinação utilizadas foram: faixa de temperatura de $30^{\circ} \mathrm{C}$ a $450^{\circ} \mathrm{C}$, a uma razão de aquecimento de $10^{\circ} \mathrm{C} / \mathrm{min}$, durante um tempo de 4 horas.

A peneira molecular mesoporosa MCM-41 foi obtida partindo de sílica gel e silicato de sódio como fontes de silício e sódio, brometo de cetiltrimetilamônio como direcionador estrutural e água deionizada. Para obtenção do material foi utilizado um gel de síntese com a seguinte composição molar: 4,730 CTMABr: 1,914 $\mathrm{Na}_{2} \mathrm{O}: 2,472 \mathrm{SiO}_{2}: 45,248 \mathrm{H}_{2} \mathrm{O}$. A síntese foi realizada em duas etapas, consistindo no preparo de duas soluções. A solução 1 contém fonte de silício e sódio e metade da água destilada utilizada para a síntese. A solução 2 contém o direcionador CTMABr e a outra metade da água destilada necessária para síntese. A primeira solução foi preparada mantendo-se a mistura sob agitação constante a $60^{\circ} \mathrm{C}$ por duas horas. A segunda solução foi mantida sob agitação durante 30 minutos a temperatura ambiente. Após duas horas de agitação, a solução 1 foi adicionada sobre a solução 2, mantendo-se a mistura sob agitação por 30 minutos em temperatura ambiente, resultando na obtenção do gel de síntese. Após a preparação do gel o mesmo foi colocado em autoclave de teflon revestida com aço e em seguida o conjunto foi levado à estufa a $100^{\circ} \mathrm{C}$ por 72 horas. A cada 24 horas foram realizadas correções do $\mathrm{pH}$ do sistema para entre 9-10 com solução de ácido acético a 30\% (Figura 3). Após esse tempo o produto foi retirado da autoclave e filtrado à vácuo, seguido de lavagem com água destilada e secagem em estufa a $100^{\circ} \mathrm{C}$ por 2 horas.

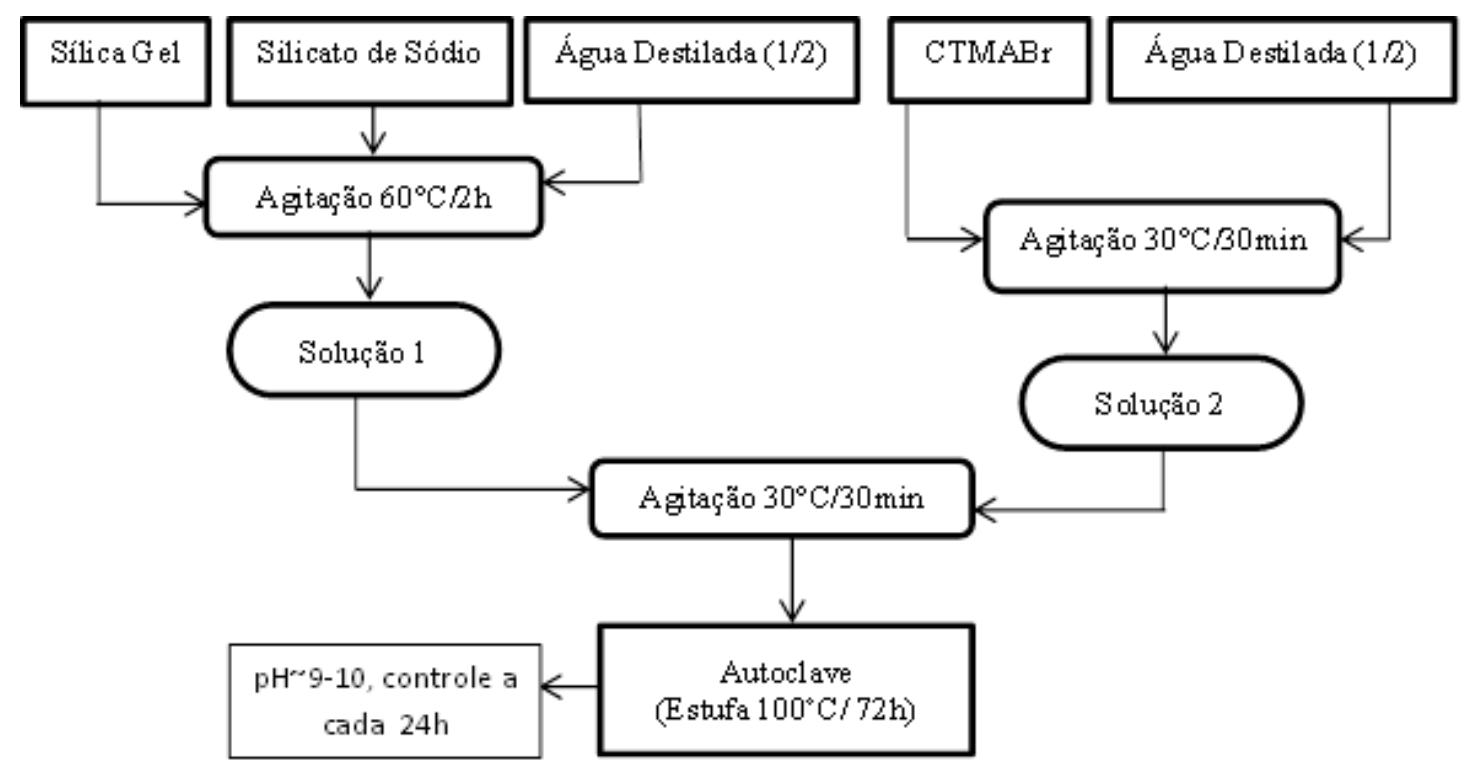

Figura 3: Principais etapas da síntese do MCM-41.

A obtenção do catalisador micro-mesoporoso do tipo HZSM-5/MCM-41 foi via mecanosíntese. Primeiramente foram pesadas as massas do material microporoso (HZSM-5) e do mesoporoso (MCM-41), os quais foram previamente secos durante 1 hora em uma estufa a uma temperatura de $100^{\circ} \mathrm{C}$. As proporções mássicas do material microporoso e mesoporoso foram estabelecidas como 1:4, ou seja, $20 \%$ de zeólita microporosa HZSM-5 e $80 \%$ do material mesoporoso (MCM-41). Após as pesagens dos materiais microporoso e mesoporoso, estes foram misturados através da moagem num almofariz durante 20 minutos até homogeneização. A moagem foi realizada em quatro etapas: uma quantidade equivalente a $1 / 4$ da massa do material microporoso e $1 / 4$ do material mesoporoso foi espalhado no almofariz e submetido à moagem manual contínua durante 5 minutos. 
Este procedimento foi repetido por mais de três vezes, sendo que a cada etapa a massa dos materiais microporo e mesoporoso foi adicionado sobre o material já moído contido no almofariz.

A obtenção do catalisador compósito micro-mesoporoso do tipo La/HZSM-5/MCM-41foi através do processo de impregnação do material HZSM-5/MCM-41com óxido de lantânio através do método de impregnação por via úmida seguindo a metodologia adaptada [11].

\section{2- Caracterização}

\subsection{1- Difratogramas de raios-X}

Todos os catalisadores sintetizados foram caracterizados via difratometria de raios-X. Para os materiais sintetizados, o principal objetivo era verificar se ocorreu a formação ou manutenção da estrutura desejada. Enquanto que para os materiais impregnados com terra rara o objetivo da análise era verificar se a integridade da estrutura se manteve. Os difratogramas foram obtidos com radiação $\mathrm{CuK} \alpha(\lambda=1,5406 \mathrm{~nm})$ e $2 \theta$ na faixa de 1,5 a $10^{\circ}$, sendo o passo de varredura de $0,020^{\circ}$ min. Padrões de difração de raios- $X$ que servem para caracterizar diferentes tipos de zeólitas podem ser encontrados na literatura [12].

\subsection{2- Avaliação catalítica}

A fim de avaliar o potencial dos catalisadores micro-mesoporosos os mesmos foram submetidos à reação modelo de craqueamento de isopropilbenzeno em um microreator catalítico de leito fixo com fluxo contínuo. O microreator utilizado consistiu de um tubo de aço inox 316, com comprimento de $30 \mathrm{~cm}$ e $0,635 \mathrm{~cm}$ de diâmetro interno (1/4 $)$. De modo a minimizar as limitações difusionais internas ao transporte de massa, durante a preparação do leito, 300,0 $\mathrm{mg}$ de cada amostra de catalisador foram utilizadas na forma de um fino pó e este foi colocado entre duas camadas de lã de quartzo alocadas de forma simétrica à distância longitudinal do reator com referência a sua entrada e saída. Foram também levados em consideração, os seguintes aspectos: reação isotérmica em leito fixo, fase vapor em estado de gás ideal, escoamento em fluxo pistonado, porosidade uniforme e queda de pressão no leito desprezível.

As condições reacionais do craqueamento catalítico do isopropilbenzeno (molécula modelo) foram determinadas em estudos prévios com isopropilbenzeno e com outras moléculas modelos [13, 14]. Antes do início das reações, após a colocação da amostra de catalisador no reator, as mesmas foram ativadas com aquecimento in situ em fluxo de nitrogênio de $50 \mathrm{~mL} \mathrm{~min}^{-1}$ partindo da temperatura ambiente até a temperatura de reação $\left(450^{\circ} \mathrm{C}\right) \mathrm{com}$ taxa de aquecimento de $5^{\circ} \mathrm{C} \mathrm{min}^{-1}$. Após alcançar essa temperatura o reator foi isolado, desviando o fluxo pelo by pass e nesse momento foi acionada a bomba que impulsionava uma seringa, contendo isopropilbenzeno líquido pelas linhas capilares de fluxo da unidade. A seringa de injeção de líquido tem um volume interno de $20 \mathrm{~mL}$ e é construída em aço inox, possuindo orings de vedação de Chemrez. O líquido foi injetado pela seringa a $50 \mu \mathrm{L} \mathrm{min}{ }^{-1}$ e foi sendo continuamente vaporizado e transportado pelas linhas de fluxo de forma contínua a $200^{\circ} \mathrm{C}$ e se misturando com a corrente de gás inerte (nitrogênio) durante 10 minutos até que as linhas ficassem totalmente preenchidas da mistura, sendo direcionados após esse tempo ao reator em fluxo descendente a uma WHSV (Weight Hourly Space Velocity) de $3 \mathrm{~h}^{-1}$. Os gases efluentes do reator foram injetados em intervalos de tempo de aproximadamente 3,5 minutos, através de uma válvula de amostragem Valco de 6 vias, em um cromatógrafo a gás Agilent $6890 \mathrm{~N}$ equipado com uma coluna HP-5 - 5\% de Phenyl Methyl Siloxane de $30 \mathrm{~m}$. A análise dos produtos foi realizada através do detector FID à temperatura de $300^{\circ} \mathrm{C}$ com fluxo na coluna de capilar $1 \mathrm{~mL} \cdot \mathrm{min}^{-1}$. Os cromatogramas oriundos das injeções foram integrados através do software ChemStation da Agilent. 


\section{RESULTADOS E DISCUSSÃO}

A Figura 4 mostra o difratograma de raios-X da zeólita ácida HZSM-5. A identificação das fases presentes foi realizada com base na comparação do difratograma da zeólita em estudo com dados da literatura [12]. As linhas de difração mais intensas ocorrem próximas a $2 \theta=7,9^{\circ} ; 8,8^{\circ}$ e $23,1^{\circ}$. Estes picos são característicos desse tipo de material. Os demais picos presentes são atribuídos a zeólita com estrutura do tipo MFI e com alto grau de pureza.

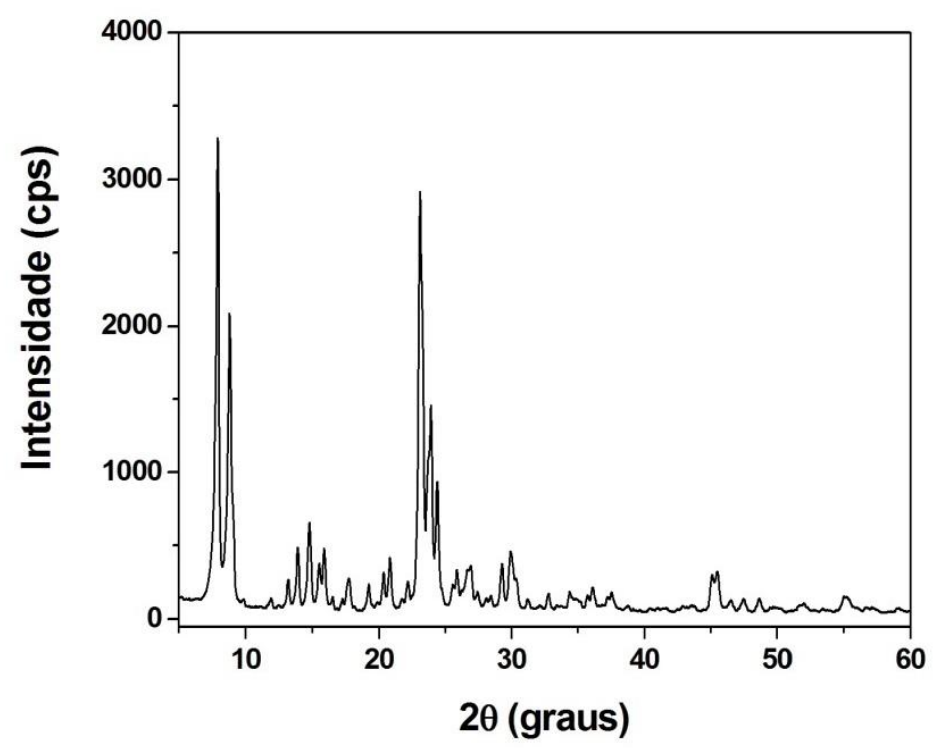

Figura 4: Difratograma de raios-X da zeólita HZSM-5.

Na Figura 5 têm-se o difratograma de raios-X do MCM-41. O padrão de DRX da amostra é típico do MCM-41 hexagonal e apresenta os picos de difração referentes aos planos (100), (110) e (200) localizados em valores de $2 \theta$ próximos a $2,2^{\circ}, 3,6^{\circ}$ e $4,2^{\circ}$, respectivamente.

$\mathrm{O}$ valor do parâmetro mesoporoso $\left(\mathrm{a}_{0}\right)$ para o MCM-41 foi calculado de acordo com a expressão $\mathrm{a}_{0}$ $=2 \mathrm{~d}_{(100)} / 3^{0,5}$, onde $\mathrm{d}_{(100)}=$ distância interplanar dos planos (100). $\mathrm{O}$ valor do parâmetro mesoporoso (a) para o MCM-41 apresentado na Tabela 1 é similar aos encontrados na literatura [10].

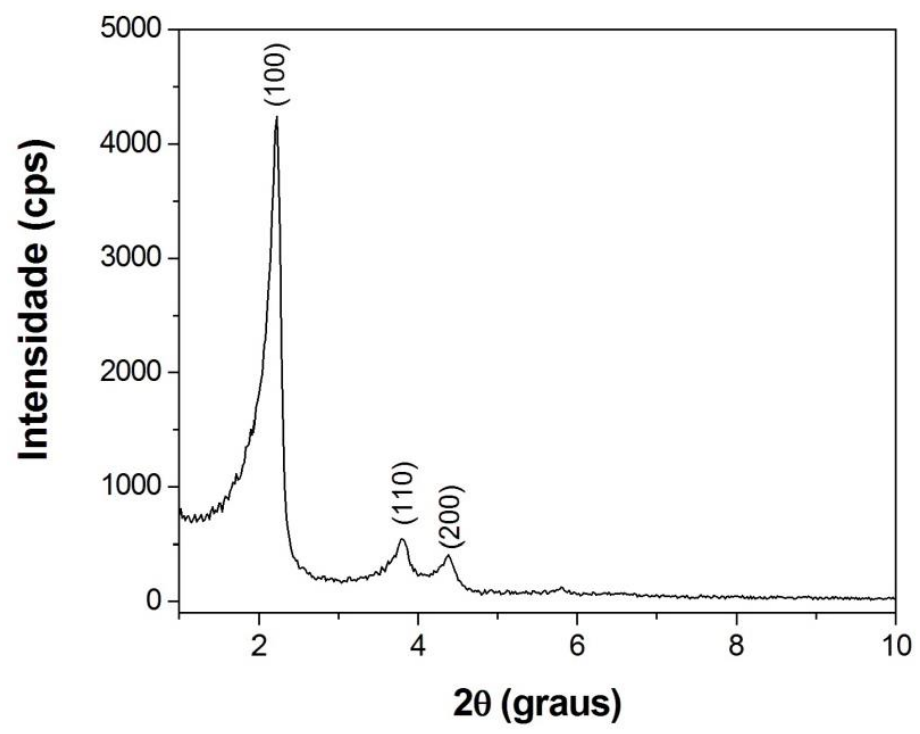

Figura 5: Difratograma de raios-X do MCM-41. 
Na Figura 6 têm-se os difratogramas de raios-X dos catalisadores compósito micro-mesoporosos do tipo HZSM-5/MCM-41 e La/HZSM-5/MCM-41. Para comparação, o difratograma do MCM-41 e do La/MCM-41 também é apresentado na Figura. Todos os difratogramas dos catalisadores micromesoporosos (Figuras 6c e 6d) apresentaram picos de difração devido ao MCM-41 em baixos ângulos $\left(2 \theta \approx 2,2^{\circ}, 3,6^{\circ}\right.$ e $\left.4,2^{\circ}\right)$, confirmando desta forma a manutenção da estrutura do MCM-41 no catalisador micro-mesoporoso. Os catalisadores compósitos HZSM-5/MCM-41 e La/HZSM-5/MCM41 também mostraram alguns picos característicos da HZSM-5 em ângulos $2 \theta=7,9$ e $8,8^{\circ}[12,15]$.

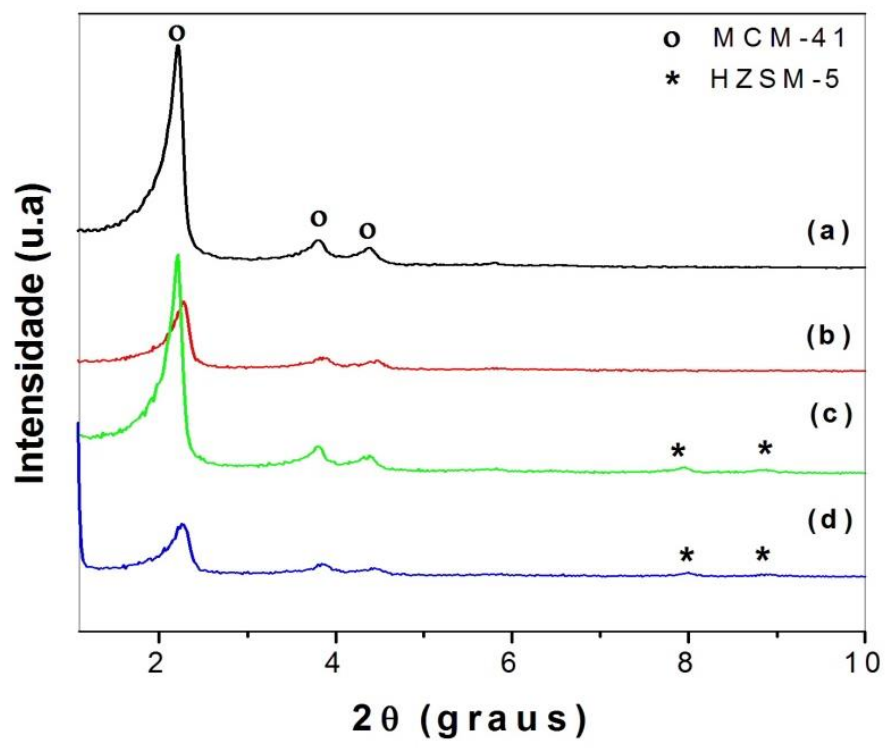

Figura 6: Difratograma de raios-X dos catalisadores: (a) MCM-41, (b) La/MCM-41, (c) HZSM-5/MCM-41 e (d) $L a / H Z S M-5 / M C M-41$.

O grau de ordenamento (GO) para o MCM-41 foi calculado com relação ao pico (100) do MCM-41 e comparando-se a intensidade deste pico no MCM-41 puro com relação às respectivas intensidades nas demais amostras. Os valores do parâmetro mesoporoso $\left(\mathrm{a}_{0}\right)$ e o grau de ordenamento para o MCM-41 nos catalisadores La/MCM-41, HZSM-5/MCM-41 e La/HZSM-5/MCM-41 estão apresentados na (Tabela 1). De acordo com o apresentado na (Figura 6) é possível observar que a intensidade dos picos relativos ao MCM-41 é ligeiramente diminuída nas amostras La/MCM-41 e La/HZSM-5/MCM-41 em relação ao MCM-41 puro. Isto é refletido na diminuição do grau de ordenamento da estrutura. Além disto, também é possível observar que ocorreu nestas amostras o deslocamento desse pico para ângulos um pouco maiores em $2 \theta$ (Tabela 1), o que é demonstrado através da ligeira redução nos valores do parâmetro mesoporoso nas amostras La/MCM-41 e La/HZSM-5/MCM-41. Estes aspectos podem estar relacionados à estabilidade hidrotérmica do material, que pode ter sido afetada durante os procedimentos de síntese, assim como a condensação dos grupos silanóis e contração da rede do MCM-41. Apesar disto, pode-se concluir que esta redução no valor do parâmetro mesoporoso não é significativa, e que a presença dos três picos característicos do MCM-41 é um indicativo que os mesocanais são mantidos [10].

Tabela 1: Principais parâmetros estruturais do MCM-41 nos catalisadores.

\begin{tabular}{cccccc}
\hline Catalisador & $\begin{array}{c}\text { Índice de } \\
\text { Miller (hkl) }\end{array}$ & $\mathbf{2 \theta}$ (graus) & $\mathbf{d}_{\mathbf{1 0 0}}(\mathbf{n m})$ & $\mathbf{a}_{\mathbf{0}}(\mathbf{n m})$ & $\mathbf{G O}(\boldsymbol{\%})$ \\
\hline MCM-41 & $(100)$ & 2,22 & 3,98 & 4,60 & 100 \\
La/MCM-41 & $(100)$ & 2,28 & 3,88 & 4,48 & 31 \\
HZSM-5/MCM-41 & $(100)$ & 2,22 & 3,99 & 4,60 & 97 \\
La/HZSM-5/MCM-41 & $(100)$ & 2,27 & 3,90 & 4,50 & 24 \\
\hline
\end{tabular}


A Figura 7 mostra os resultados dos testes catalíticos de craqueamento de cumeno em função do tempo de reação usando os catalisadores micro-mesoporosos La/HZSM-5/MCM-41 (Figura 7a) e HZSM-5/MCM-41 (Figura 7b). Para efeito de comparação, os resultados dos testes catalíticos dos catalisadores HZSM-5, MCM-41, La/HZSM-5 e La/MCM-41 também são apresentados.

Conforme pode ser observado na Figura 7, a conversão do cumeno sobre os catalisadores se mantém praticamente constante em função do tempo de reação, exceto para os catalisadores La/HZSM-5 e La/MCM-41 cuja conversão diminui lentamente em função do tempo de reação.

De acordo com os resultados pode-se observar que o catalisador La/HZSM-5/MCM-41 obtido nesse estudo mostrou uma maior conversão do cumeno em relação aos demais catalisadores, e que a presença do lantânio promoveu um ligeiro aumento nos valores de conversão para este catalisador em comparação ao catalisador HZSM-5/MCM-41. Em geral a escala de atividade variou na sequência: La/HZSM-5/MCM-41 > HZSM-5/MCM-41 > MCM-41 > La/HZSM-5 > HZSM-5 > La/MCM-41 e os níveis de conversão na faixa de 3 a $7 \%$.
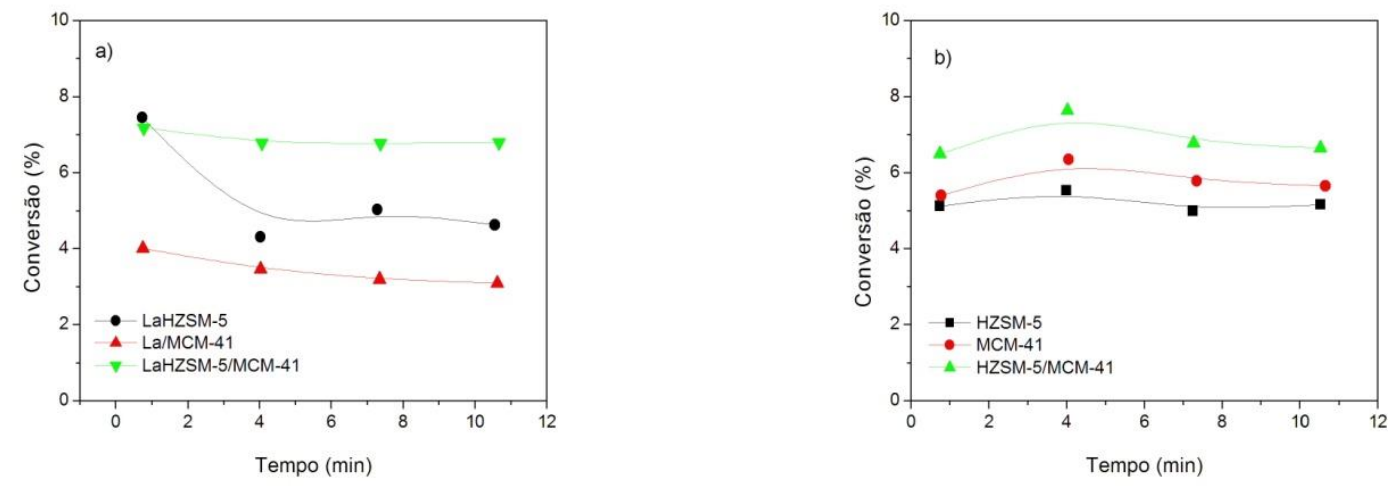

Figura 7: Conversão do cumeno sobre catalisadores: (a) La/HZSM-5, La/MCM-4le La/HZSM-5/MCM-41 e (b) HZSM-5, MCM-41 e HZSM-5/MCM-41.

Embora os catalisadores HZSM-5/MCM-41, MCM-41, La/HZSM-5/MCM-41 e La/MCM-41 tenham apresentado valores do parâmetro mesoporoso muito próximos, os valores do grau de ordenamento variou significativamente para cada catalisador. Apesar das diferenças nestas características não houve diferenças muito expressivas no desempenho dos catalisadores em relação a estas características. O catalisador La/HZSM-5/MCM-41 que apresentou menor grau de ordenamento hexagonal mostrou a maior atividade catalítica.

$\mathrm{O}$ efeito da presença do lantânio nos catalisadores também pode ser observado nos resultados apresentados na Figura 7a. Em geral o lantânio é inserido na composição do catalisador para melhorar sua atividade, seletividade e estabilidade. De uma forma geral, a inserção do lantânio provocou um ligeiro aumento na conversão, exceto para amostra La/MCM-41 que foi observado um comportamento contrário. Neste caso, isto pode estar correlacionado com um maior bloqueio do sistema de poros do MCM-41 devido à presença do lantânio.

O efeito do componente zeolítico microporoso (HZSM-5) também pode ser observado. Embora a zeólita HZSM-5 deva apresentar uma acidez significativamente maior do que o MCM-41, em geral foi observado uma atividade menor para os dois catalisadores que não contém o componente mesoporoso e contém apenas o componente zeólitico (HZSM-5 e La/HZSM-5) do que o MCM-41. Isto sugere que possa estar ocorrendo alguma restrição difusional para evolução do cumeno nos poros da zeólita. Ou seja, como o diâmetro cinético do cumeno é de $6,8 \AA$, pode-se inferir que esta molécula possa estar sofrendo alguma restrição difusional nas aberturas da zeólita ZSM-5, a qual tem de 5 a 5,7 $\AA$.

A atividade catalítica é bastante influenciada quando se trata de materiais com estrutura porosa devido ao transporte de espécies químicas, pois a difusividade de moléculas reagentes assim como dos produtos formados no interior do meio poroso destes materiais depende do diâmetro dos poros. Moléculas maiores com o diâmetro próximo ao diâmetro de poro têm sua difusão prejudicada, ou 
restrição difusional, consequentemente, um acesso difícil aos sítios ativos do catalisador e produtos volumosos pode ficar retido ou ter sua formação desfavorecida $[13,16]$.

Estes resultados sugerem que o cumeno não sofre limitações difusionais no componente mesoporoso (MCM-41) do catalisador e que a conversão é influenciada pelo efeito sinergético entre o componente mesoporoso, o componente microporoso zeolítico, e a presença de lantânio.

Os produtos típicos das reações de conversão do cumeno em condições típicas de craqueamento catalítico são propeno e benzeno [14,17], sendo o propeno o principal produto formado por essa reação. A proporção teórica de formação de propeno e benzeno é de 1:1, de acordo com a proposta de mecanismo para o craqueamento do cumeno (Figura 8). Porém pode haver formação de outros produtos em quantidades bem reduzidas, podendo estes serem formados por reações secundárias quando se tem a presença de catalisadores com força ácida elevada [14].

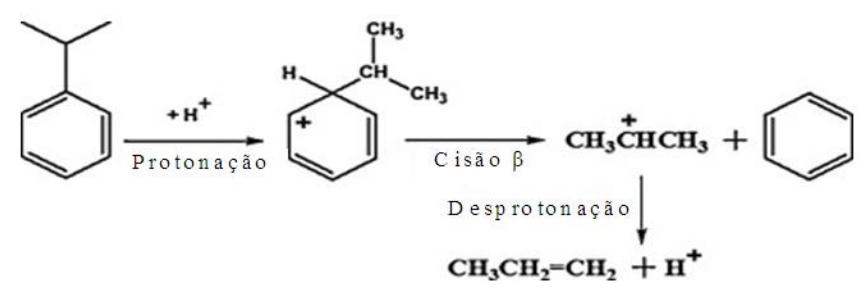

Figura 8: Mecanismo de reação do craqueamento catalítico do cumeno produzindo propeno e benzeno, adaptado de [14].

Na Figura 9 são apresentados os resultados de produção de propeno após o craqueamento catalítico do cumeno.

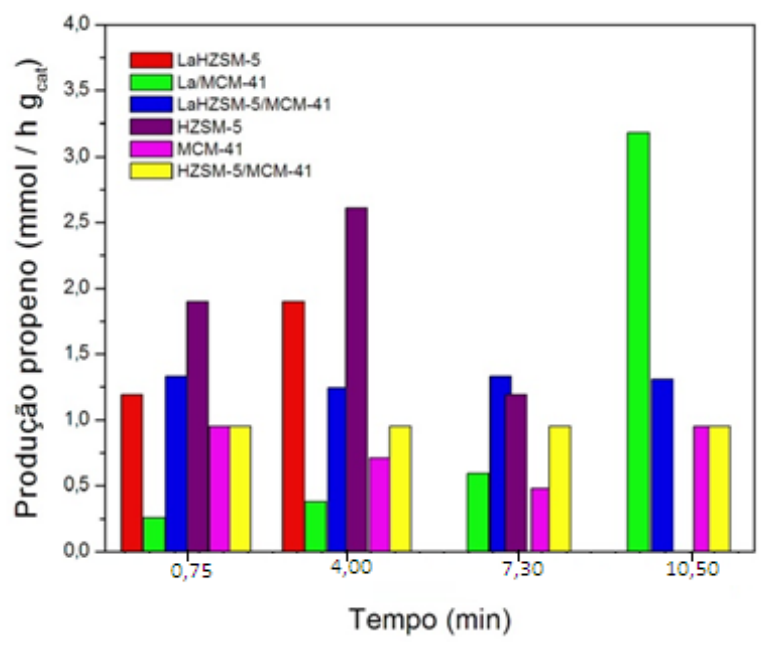

Figura 9: Produção de propeno sobre os catalisadores HZSM-5, MCM-41, HZSM-5/MCM-41, La/HZSM-5, La/MCM-41 e La/HZSM-5/MCM-41.

A Figura 9 mostra que os catalisadores micro-mesoporosos La/HZSM-5/MCM-41 e HZSM5/MCM-41 são os únicos que tem uma produção de propeno relativamente constante, enquanto que para os demais esta oscila significativamente. Os valores de produção de propeno para estes dois catalisadores são intermediários, sendo que para o catalisador La/HZSM-5/MCM-41 esta foi ligeiramente maior do que para o catalisador HZSM-5/MCM-41. 
Para menores tempos de reação, a zeólita (HZSM-5 e La/HZSM-5) mostrou resultados mais expressivos de produção de propeno quando comparado aos demais catalisadores, porém com o aumento do tempo de reação e queda na atividade do catalisador esta reduziu drasticamente. Por outro lado, em menores tempos de reação, o MCM-41 e La/MCM-41 mostraram valores de produção de propeno relativamente menores do que o da zeólita. Porém, com o aumento do tempo de reação, o valor de produção de propeno cresceu para o La/MCM-41 e para o MCM-41. O crescimento foi muito mais expressivo para o La/MCM-41. Uma elevada produção de propeno pode não ser um resultado interessante quando este atua na geração de coque e desativa severamente o catalisador.

Embora os catalisadores La/HZSM-5/MCM-41 e HZSM-5/MCM-41 tenham gerado valores intermediários de produção de propeno, o fato destes valores terem sido constantes e terem apresentados maiores valores de conversão, pode-se concluir com base nestes aspectos, que estes foram os melhores catalisadores para a produção de propeno.

Estes resultados sugerem que a produção de propeno também é influenciada pelo efeito sinergético entre o componente mesoporoso, o componente microporoso zeolítico, e a presença de lantânio.

\section{CONCLUSÃO}

Os difratogramas de raios-X dos catalisadores HZSM-5/MCM-41 e La/HZSM-5/MCM-41 mostraram a presença das fases microporosa (HZSM-5) e mesoporosa (MCM-41) em conjunto. O catalisador La/HZSM-5/MCM-41 apresentou uma sutil redução no parâmetro do mesoporo e uma significativa redução no grau de ordenamento hexagonal em relação ao MCM-41 puro, enquanto que o catalisador HZSM-5/MCM-41 não apresentou estas reduções.

Os resultados mostraram que o catalisador La/HZSM-5/MCM-41 foi o que obteve resposta mais favorável frente às reações de craqueamento catalítico, com maior nível de conversão do cumeno e melhores resultados de produção de propeno quando comparado aos demais catalisadores.

Os resultados sugerem que a conversão e a produção de propeno são influenciadas pelo efeito sinergético entre o componente mesoporoso, o componente microporoso zeolítico, e a presença de lantânio.

\section{AGRADECIMENTOS}

Os autores agradecem ao Programa de Pós-graduação em Engenharia Química da UFS (PEQ/UFS), ao Conselho Nacional de Desenvolvimento Científico e Tecnológico (CNPq) e a Coordenação de Aperfeiçoamento de Pessoal de Nível Superior (CAPES) pelo apoio financeiro concedido e bolsa concedida.

\section{REFERÊNCIAS BIBLIOGRÁFICAS}

1. Tominaga H, Tameki M. Chemical reaction and reactor design. Londres: John Wiley \& Sons; 1997. 403p.

2. Von Ballmoss R, Harris DH, Magee JS. Handbook of Heterogeneous Catalysis. Weinheim: John Wiley \& Sons; 1997. 1955p.

3. Lansarin MA. Modelagem e simulação de seção de conversão de uma unidade FCC [Tese de doutorado em Engenharia Química]. Campinas (SP): Universidade Estadual de Campinas em São Paulo; 1997.149p.

4. Pinheiro CIC, Fernandes JL, Domingues L, Chambel AJS, Graça I, Oliveira NMC, Cerqueira HS, Ribeiro F R. Fluid Catalytic Cracking (FCC) Process modeling, simulation, and control. Ind. Eng. Chem. Res. 2012; 51 (1): 1-29, doi: 10.1021/ie200743c.

5. McDaniel CV, Maher PK, US Patents 3392 192,1966.

6. Dwyer J, Rawlence DJ. Fluid catalytic cracking: chemistry. Catal. Today. 1993; 18(4):487-507, doi:10.1016/0920-5861(93)80065-9.

7. Gianeto GP. Zeolitas: características, propiedades y aplicaciones industriales. Caracas: Facultad de Ingeniería (UCV); 2000. 351p.

8. Scherzer J. Octane - enhancing zeolite FCC catalysts. Nova York: CRC Press; 1990. 253p. 
9. Baerlocher CH, McCusker LB, Olson DH. Atlas of zeolite framework types. New York: Elsevier; 2007. 398p.

10. Kresge CT, Roth WJ. The discovery of mesoporous molecular sieves from the twenty year perspective. Chem. Soc. Rev. 2013; 42: 3663-3670, doi: 10.1039/C3CS60016E.

11. Oliveira TG. Desenvolvimento de peneiras moleculares mesoporosas do tipo MCM-41 e MCM-48 impregnadas com aminas para utilização na adsorção de $\mathrm{CO}_{2}$. [Dissertação de Mestrado em Química]. São Cristóvão (SE): Universidade Federal de Sergipe em Sergipe; 2012. 123p.

12. Treacy MMJ, Higgins JB. Collection of Simulated XRD powder patterns for zeolites. Amsterdam: Elsevier; 2001.379p.

13. Pujro RA, Falco MG, Pedrosa AMG, Souza MJB, Morgado Jr. E, Sedran U. Yield of aromatics from naphthenics upon catalytic cracking. J. Braz. Chem. Soc. 2012; 23: 1378-1387, doi: 10.1590/S010350532012000700023

14. Annuar NHR, Jalil AA, Triwahyono S, Fatah NAA, Mamat CR. Cumene Cracking over chromium oxide zirconia: Effect of chromium (VI) oxide precursors. Appl. Catal. A Gen. 2014; 475: 487-496, doi:10.1016/j.apcata.2014.02.005.

15. Na J, Liu G, Zhou T, Ding G, Hu S, Wang L. Synthesis and catalytic performance of ZSM-5/MCM-41 zeolites with varying mesopore size by surfactant-directed recrystallization. Catal. Lett. 2013; 143(3): 267275, doi: 10.1007/s10562-013-0963-0.

16. Li N, Zhang YY, Chen L, Au CT, Yin SF. Synthesis and application of HZSM-5-silicalite-1 core-shell composites for the generation of light olefins from $\mathrm{CH}_{3} \mathrm{Br}$. Microp. Mesop. Mater. 2016; 227: 76-80, doi:10.1016/j.micromeso.2016.02.017.

17. Ribeiro FM, Pedrosa, AMG, Souza MJB. Conversion of Isopropylbenzene over AlSBA-15 Nanostructured Materials. Modern Res. Catal. 2014; 03: 94-98, doi: 10.4236/mrc.2014.33012. 